\title{
Automatic Segmentation of Hippocampus and Classification of brain MRI for Alzheimer's Detection
}

\author{
Dulumani Das ${ }^{1}$, Sanjib Kumar Kalita ${ }^{2}$ \\ ${ }^{1,2}$ Department of Computer Science, Gauhati University, Guwahati, India, ${ }^{1}$ dulumonids1@ gmail.com, \\ ${ }^{2}$ sanjib959@gauhati.ac.in
}

\begin{abstract}
Hippocampus is the structure of brain that is mostly affected by Alzheimer's disease at an early stage. Atrophy of hippocampus has been found as a predictive feature for Alzheimer's disease diagnosis. To measure the atrophy of hippocampus we need to segment it out from surrounding structures of brain. Manual segmentation of hippocampus has been found standard technique for hippocampus segmentation in literature, but is very time consuming and depends on particular anatomical information. In this work we have proposed an automatic approach to segment hippocampus considering texture and active contour from the brain Magnetic Resonance Image. After segmentation, features based on atrophy and shape of hippocampus has been measured. Support vector machine classifier with radial basis function kernel has been analyzed with extracted features for classification of Alzheimer's and control subjects. In the proposed technique, $200 \mathrm{AD}$ MRI and 200 control MRI have been considered from Alzheimer's Disease Neuroimaging Initiative database. The experiment have shown 93\% accuracy, 0.96 sensitivity and 0.90 specificity with atrophy feature and $94 \%$ accuracy, 0.96 sensitivity and 0.92 specificity with shape feature. Further, 0.96 sensitivity, 1 specificity and $98 \%$ accuracy have been obtained with the fusion of atrophy and shape feature.
\end{abstract}

Key words: Alzheimer's disease, Hippocampus, Segmentation, Support Vector Machine.

\section{INTRODUCTION}

Alzheimer's disease (AD) is a progressive disease of brain that causes atrophy of different parts of brain. This is the main cause of dementia in old age people. According to Alzheimer's Association of India more than 4 million people have dementia. AD starts slowly but gradually shrinks the brain cells and become worse over time. The common early sign of $\mathrm{AD}$ is difficulty in remembering things or recent conversation or recent events. These early signs are often ignored as normal sign of aging. There is no cure of $\mathrm{AD}$ as it is irreversible disease but the progress of the disease can be slowed down with fast diagnosis at early stage. Recent developments of medical imaging technologies make it somewhat possible to diagnose $\mathrm{AD}$ by analysing the information obtained from medical images. Magnetic Resonance Imaging (MRI) is one of the most commonly used medical imaging techniques for $\mathrm{AD}$ diagnosis.

Hippocampus of brain is the structure that is early affected by AD. Different features based on hippocampus have been found in literature as predictive markers for $\mathrm{AD}$ detection. It is a challenging task to segment hippocampus from brain MRI with accurate accuracy due to its deformed boundary, complex shape, interconnected structures of brain and variability. There are number of research works on automatic hippocampus segmentation have been reported in literature. Based on literature automatic hippocampus segmentation techniques can categorized as atlas based method, deformable model , classification based and combination of these mentioned methods. In atlas based method ([1], [2], [3], [4], [5]), an atlas is an image which is selected either randomly or by examining different images of the dataset. The region of interest (hippocampus) is segmented manually in the atlas and is considered as ideal of the dataset for segmentation. Then the atlas is registered with the image to be segmented to align both the images. After registration the hippocampus region of the atlas is transferred to the target image. Atlas based method has been further enhanced to multi atlas ([6], [7]) and probabilistic atlas ([2], [3]). In multi atlas method, instead of single atlas, multiple atlases are used for registration with the target image. Segmented structures obtained from multiple atlases are then combined to obtain the final segmented structure of the target image. There are different label fusion techniques that have been used to combine multiple atlases. In case of probabilistic atlas method different atlases are registered with one another to obtain statistical information (shape, texture) of the hippocampus and then obtained information has been given as input to other segmentation model such as deformable model or classification model for final segmentation of hippocampus. The result of atlas based technique also depends on registration technique being used. A deformable model is one most commonly used techniques for hippocampus segmentation ([8], [9], [10]). Deformable model depicts a group of techniques that characterizes the variability in the shape or texture of an image object by delineating the object's outline. The simplest deformable model, widely used for hippocampus segmentation is active contour model ([8], [11]). In active contour model an initial contour is placed in the region of interest of the image and then is deformed in each iteration by internal and external energies generating new contours. The internal energy controls the deformations made and external energy controls the fitting of the contour onto the image object. Active shape 
model ([9], [12]) is an extension of active contour model. In active shape model, constraints that are generated from prior knowledge have been used to modify the internal energy, so that the deformation is limited to a shape range of the segmented object. Active appearance model ([13], [14]) is another deformable method that is modelled by modifying active shape model. In this model constraints were incorporated in external energy to obtain a better fit of the contour onto the segmented object. The literature review reveals that atlas based method and deformable model have been used in combination for hippocampus segmentation ([2], [15]).

Classification based methods for hippocampus segmentation, that have been found on literature includes artificial neural network, random forest, support vector machine ([16], [17], [18]).

A number of works on $\mathrm{AD}$ detection have been reported in literature during the last decade. The classifiers that have been used for $\mathrm{AD}$ detection commonly includes support vector machine ([19], [20], [21], [22], [23]), artificial neural network (ANN) [24], linear discriminant analysis (LDA) [25], deep neural network [26], k nearest neighbor (KNN) [27], whereas support vector machine has been found as one of the most widely used techniques.

\section{DATASET USED}

The dataset used in this work has been obtained from the Alzheimer's Disease Neuroimaging Initiative (ADNI) database (adni.loni.usc.edu).The ADNI was launched in 2003 as a public-private partnership, led by principal investigator Michael W. Weiner, MD. The primary goal of ADNI has been to test whether serial magnetic resonance imaging (MRI), positron emission tomography (PET), other biological markers, and clinical and neuropsychological assessment can be combined to measure the progression of mild cognitive impairment (MCI) and early Alzheimer's disease (AD). Among the three views of MRI i.e. axial, coronal and sagittal, the coronal view of T1 weighted MRI from ADNI screening 1.5T database has been considered for experiment. 200 control MRI and 200 AD MRI of age group 55-85 containing both male and female patients have been taken for experiment. The images considered were already pre-processed images for correction of image geometry distortion and image intensity non-uniformity by ADNI. All the images have been resized to $400 \times 400$ pixels.

\section{HIPPOCAMPUS SEGMENTATION}

Hippocampus of brain is the structure that is responsible for formation and storage of memory and is the part early and mostly affected by AD. This is the reason that AD affected people faces difficulty in remembering things. In diagnosis of $\mathrm{AD}$ hippocampus plays an important role as atrophy of hippocampus is a predictive marker for diagnosis. In this work an automatic approach for hippocampus segmentation has been proposed. Hippocampus segmentation has been performed after pre processing step that includes contrast enhancement [28] and skull stripping (Removal of unwanted portions like skull, neck etc. from brain). For hippocampus segmentation initially one AD image has been selected as reference image. We have performed texture analysis using standard deviation filtering on the pre processed reference image. Due to AD hippocampus of the brain get shrink causing black holes in that region. So standard deviation filtering gives high values for hippocampus region of brain compared to other parts due to high variability of pixel values. This is the actual region of selecting an AD image as reference image. Although, some other part of brain MRI can show high value for standard deviation filtering, we can separate out the hippocampus region as the brain has two hippocampus at same position on left and right side. If we extract out the regions that shares maximum number of common rows and also have high value of standard deviation filtering then we get the left hippocampus region and right hippocampus region. Initially, we have created two rectangular masks, one for left hippocampus and one for right hippocampus from the reference image. Further we have created two more masks each for left and right hippocampus by increasing and decreasing the size of the initial mask and name them as large mask and small mask respectively. The initial mask is named as medium mask. Three types of masks have been created as there are variations in brain size. After creation of masks the target image to be segmented has been registered with the reference image using affine transformation method and then transfer the masks to the target image one by one. The mask that covers the whole hippocampus region is kept and others are discarded. Finally, active contour technique has been used on the mask for final segmentation of hippocampus. The step wise method of hippocampus segmentation has been described in Figure 1.

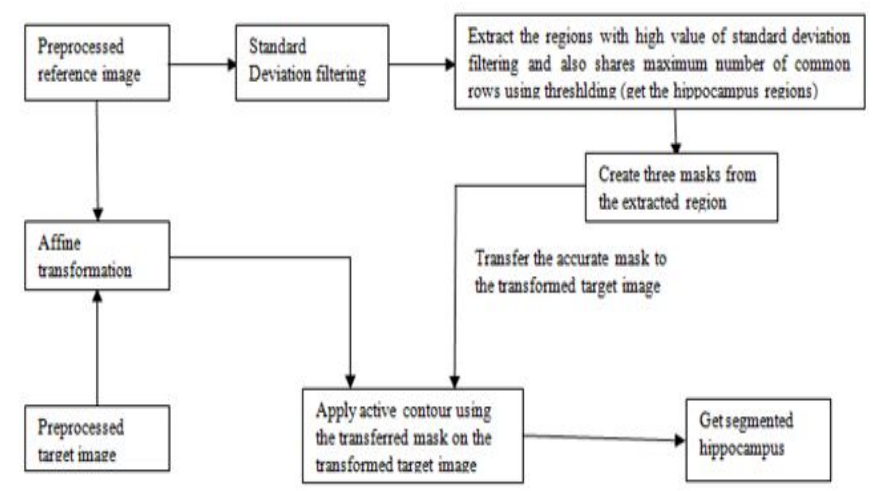

Figure 1: Step wise Method of Hippocampus Segmentation from Brain MRI

Figure 2 has described the proposed algorithm for mask creation. Mask1 and Mask2 are initially created mask. Two more masks for each hippocampus have been created by increasing and decreasing the size of the initial mask. The proposed algorithm for hippocampus segmentation and obtained results has been given in Figure 3 and Figure 4 
respectively.

Input: Pre-processed AD MRI image I (reference image).

Output: Masks for left hippocampus and right hippocampus of brain.

1. $\mathrm{I} 1=$ standard deviation filtering $(\mathrm{I})$.

2. I2= thresholding (I1). (using Otsu's technique)

3. Extract 10 largest connected components from I2.

4. For $\mathrm{i}=1$ to 10 (for each connected component) Find (rows(i), columns(i)).

5. for $\mathrm{i}=1: 10$

$$
\begin{gathered}
\text { for } \mathrm{j}=1: 10 \\
\quad \text { if }(\mathrm{i}==\mathrm{j}) \\
\quad \text { Continue; } \\
\text { end } \\
\text { if }(\mathrm{j}<\mathrm{i})
\end{gathered}
$$$$
\begin{aligned}
& \text { Continue; } \\
& \text { end } \\
& \text { in=intersect(rows }(i), \operatorname{rows}(j)) \text {; }
\end{aligned}
$$

end

6. Extract the two connected components i, j for which 'in' gives maximum number of values.

7. Mask1= bounding box (i). (left/right hippocampus)

8. Mask2= bounding box (j). (left/right hippocampus)

Figure 2: Proposed Algorithm for Mask Creation

Input: Pre-processed reference image I, Pre-processed target image $\mathrm{T}$ and mask obtained from algorithm described in figure 2 .

Output: Left hippocampus and Right hippocampus of target image $\mathrm{T}$.

1. Select the appropriate mask (maskl, maskr) for $\mathrm{T}$ that covers the whole hippocampus region.

2. $\mathrm{RT}=$ Affine transformation $(\mathrm{T}, \mathrm{I})$.

3. Lhippo=active contour (RT, maskl).

4. Rhippo=active contour (RT, maskr).

5. Lefthippo=RT, Righthippo=RT.

6. Lefthippo $(\sim$ Lhippo $)=0$.

7. Righthippo $(\sim$ Rhippo $)=0$.

8. Left hippocampus = thresholding $(\mathrm{RT})$. (using Otsu's thresholding)

9. Right hippocampus=thresholding (RT). (using Otsu's thresholding)

Figure 3: Proposed Algorithm for Hippocampus Segmentation

\section{FEATURE EXTRACTION}

After hippocampus segmentation atrophy and shape of hippocampus have been analysed. For measuring atrophy, initially we have calculated number of pixels in the whole hippocampus area and then apply active contour on the whole hippocampus region to get the actual hippocampus. The
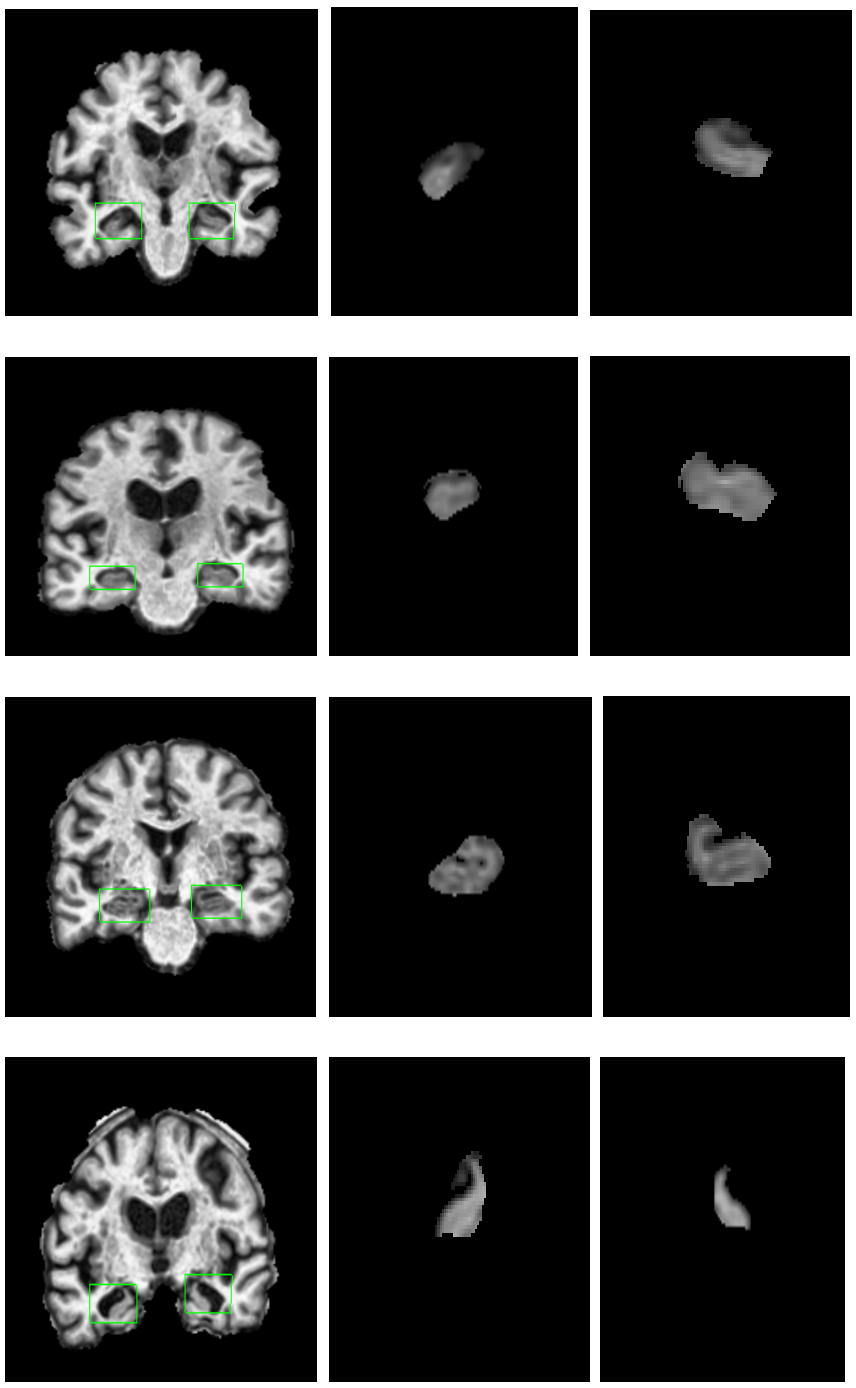

Figure 4: Results of Hippocampus Segmentation, $1^{\text {st }}$ column shows skull stripped MRI and $2^{\text {nd }}$ and $3^{\text {rd }}$ column shows corresponding segmented left and right hippocampus respectively

amount of atrophy or shrinkage has been measured by subtracting the number of pixel in actual hippocampus from the number of pixel in the whole hippocampus. The difference in atrophy of $\mathrm{AD}$ hippocampus and normal hippocampus can be analysed from the graph depicted in Figure 5. It is clearly visible in Figure 5 that the amount of atrophy is very high in AD hippocampus than normal hippocampus. The atrophy is higher than 300 for $\mathrm{AD}$ hippocampus whereas, for normal hippocampus it is lower than 300 .

The shape of segmented hippocampus has been analysed using local energy based shape histogram (LESH). LESH has been found effective for shape analysis in other application areas. So we have experimented it for our work. LESH gives the description of underlying shape of hippocampus. It gives the underlying shape by accumulating local energy of the underlying image along several filter orientations. The LESH feature works on the basis of local energy model as described by [29]. They have given the fact that the features of an image are perceived at points of maximum phase congruency. 
According to [30] the phase congruency of a two dimensional image is calculated as (1).

$E(Z)=\frac{\sum_{n} w(Z)\left|A_{n}(Z)\left(\cos \left(\varphi_{n}(Z)-\bar{\varphi}(Z)\right)-\left|\sin \left(\varphi_{n}(Z)-\bar{\varphi}(Z)\right)\right|\right)-T\right|}{\sum_{n} A_{n}(Z)+\epsilon}$

Where, $w(Z)=$ weighting of the frequency spread, $T=$ noise cancellation factor, $A_{n}=$ amplitude of the $n^{\text {th }}$ fourier component, $\boldsymbol{\varphi}_{n}=$ phase angle of the $\mathrm{n}^{\text {th }}$ fourier component, $\epsilon=$ constant value used to avoid division by zero, $\mathrm{Z}=(\mathrm{x}, \mathrm{y})$ is the image location.

To preserve the phase information filters in symmetric anti symmetric pair must be used. For this the segmented image has been convolved with a bank of Gabor kernels at each of the 16 sub-regions and along 8 different orientations [31]. The convolution has been done using the equation (2). At each image location, for each scale and orientation, it produces a complex value comprising the output of even and odd symmetric filter that gives corresponding amplitude and phase of that pixel.

$\boldsymbol{G}_{\boldsymbol{u}, v}\left(\boldsymbol{e}_{\boldsymbol{n}}, \boldsymbol{o}_{\boldsymbol{n}}\right)=\boldsymbol{I}(\boldsymbol{Z}) * \boldsymbol{\Psi}_{\boldsymbol{u}, v}(\boldsymbol{Z})$

Where, $\mathrm{z}=(\mathrm{x}, \mathrm{y})$ represents the image position, ${ }^{\prime} *{ }^{*}=$ convolution operator, $\boldsymbol{G}_{\boldsymbol{u}, \boldsymbol{v}}\left(\boldsymbol{e}_{\boldsymbol{n}}, \boldsymbol{o}_{\boldsymbol{n}}\right)=$ convolution result of the Gabor kernel with u orientation and v scale. $\boldsymbol{\Psi}_{\boldsymbol{u}, v}(\mathrm{z})=$ Gabor wavelet kernel calculated as defined by [32]. If $\boldsymbol{e}_{\boldsymbol{n}}$ and $\boldsymbol{o}_{\boldsymbol{n}}$ denote the even and odd wavelets at a scale $\mathrm{n}$, the amplitude and phase of the transform at a given wavelet scale is given by equation (3) and (4) respectively [30]

$$
\begin{aligned}
& A_{n}(Z)=\sqrt{\left(Z * e_{n}\right)^{2}+\left(Z * o_{n}\right)^{2}} \\
& \varphi_{n}(Z)=\operatorname{atan2}\left(Z * e_{n}, Z * \mathbf{o}_{n}\right)
\end{aligned}
$$

The energy or phase congruency $\mathrm{E}(\mathrm{Z})$ has been calculated as in (1) for each orientation and then summed as a whole. The energies at each orientation have been normalized by the overall sum and scales of amplitude of individual wavelet responses at a particular location [33]. So the phase congruency (PC) is calculated as in equation (5).

$P C(Z)=\frac{\left.\sum_{0} \sum_{n} w_{0}(Z) \mid A_{n, 0}(Z)\left(\cos \left(\varphi_{n, 0}(Z)-\bar{\varphi}(Z)\right)-\left|\sin \left(\varphi_{n, 0}(Z)-\bar{\varphi}(Z)\right)\right|\right)-T_{0}\right\rfloor}{\sum_{0} \sum_{n} A_{n, 0}(Z)+\epsilon}$

Where, 0 denotes the index over orientations.

This normalized local energy measure or phase congruency has been used to describe the underlying shape of hippocampus as this energy response varies with respect to the underlying shape. A local histogram accumulating the local energy along each filter orientation for each sub region of the image has been generated. The local histograms for each sub region of the image have been concatenated to create a single histogram that preserves the relationship between different parts of hippocampus. An orientation label map has also been generated where each pixel of the image allocated with the label of the orientation at which it has largest energy across all scales. The local histogram has been generated as in equation (6). $h_{r, b}=\sum w_{r} \times E \times \delta(L-b)$

Where, $\mathrm{b}=$ the current bin, $\mathrm{L}=$ orientation label map, $\mathrm{E}=$ local energy, $\mathrm{w}=$ Gaussian weighing function centred at region $\mathrm{r}$. $\boldsymbol{w}_{\boldsymbol{r}}$ has been calculated as in equation (7).

$w_{r}=\frac{1}{\sqrt{2 \pi \sigma}} e^{\left[\left(x-r_{x o}\right)^{2}+\left(y-r_{y o}\right)^{2}\right] / \sigma^{2}}$

LESH has been generated for 16 sub-regions of the hippocampus along 8 different orientation resulting $(16 \times 8)$ 128 dimensional feature vector. The algorithm for computing LESH vector has been described in Figure 6. Figure 7 has depicted the LESH vector obtained for $\mathrm{AD}$ and control subjects.

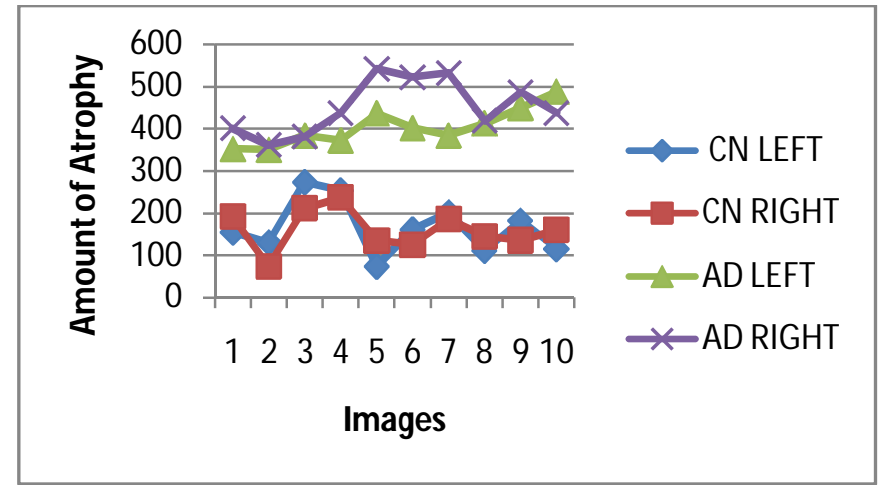

Figure 5: Difference of Atrophy between AD and Normal (CN) Hippocampus

Input: Segmented image I with a certain pixel location $\mathrm{z}=(\mathrm{x}, \mathrm{y})$

Output: LESH vector for I

1. Convolve the Fourier transform of the image I with a bank of Gabor kernels $\boldsymbol{G}_{\boldsymbol{u}, \boldsymbol{v}}\left(\boldsymbol{e}_{\boldsymbol{n}}, \boldsymbol{o}_{\boldsymbol{n}}\right)$ using (2) with u orientation and $\mathrm{v}$ scale.

2. Calculate the amplitude $\boldsymbol{A}_{\boldsymbol{n}}(\boldsymbol{Z})$ of the transform using (3).

3. Calculate the phase $\boldsymbol{\varphi}_{\boldsymbol{n}}(\boldsymbol{Z})$ of the transform using (4).

4. The energy $\boldsymbol{E}(\boldsymbol{Z})$ is calculated using (1).

5. Calculate phase congruency $\boldsymbol{P C}(\boldsymbol{Z})$ using (5).

6. Compute the LESH vector using (6) and (7).

Figure 6: Algorithm for Computation of LESH Vector
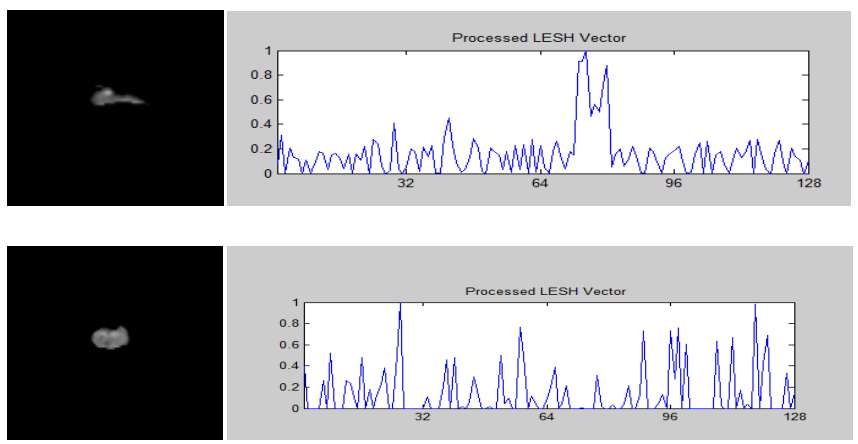

Figure 7: LESH Vector obtained for $\mathrm{AD}$ and Normal Hippocampus respectively. 


\section{CLASSIFICATION USING SUPPORT VECTOR MACHINE}

The features obtained from the section 4 have been fed to the Support vector machine for classification. It takes feature vector as input and predicts the class of each feature vector. The possible classes of this work are AD and control. It creates an optimal hyperplane that separates the two classes with the largest margin. The equation of hyperplane has been given in equation (8). As SVM is supervised classifier we need to train the model first, then the trained model can be used to predict new data.

$w^{T} \cdot l+b=0$

Where, $b=$ real number, $w=$ normal vector to the hyperplane, $1=$ feature vector.

The SVM classifier shows good results when used with appropriate kernel, based on data. Here we have used Gaussian radial basis function (GRBF) kernel. The Gaussian radial basis function kernel has been given in equation (9).

$$
k\left(l_{1}, l_{2}\right)=\exp \left(-\gamma\left\|l_{1}-l_{2}\right\|^{2}\right), \gamma>0
$$

Where, $\gamma$ is a positive parameter.

For low value of $\gamma$, points far away from the probable separation line are considered for calculation of separation line. On the other hand, in case of high value of $\gamma$, the points close to the probable separation line are considered. Tuning of this parameter has been done using 10-fold cross-validation during the training process. The value of these parameters varied logarithmically from $1 \mathrm{e}-5$ to $1 \mathrm{e} 5$.

\section{PERFORMANCE MEASURE}

In the present study, after the extraction of features from MRI brain images, the feature vectors are fed into the SVM for measuring the performance of the classifier. In this present study, fusion of atrophy and LESH feature has been performed with a view to get better accuracy. The size of the dataset used in the present study is 400 . The dataset has been split into training and testing set using hold out technique. $75 \%$ of feature vectors (300 images) of the dataset have been used for training SVM with GRBF kernel and the remaining $25 \%$ (100 images) has been used for testing the classifier. The sensitivity, specificity and accuracy of the classifier have been calculated as given in equation (10), (11) and (12) respectively.

$$
\begin{aligned}
& \text { Sensitivity }=\frac{T P}{T P+F N} \\
& \text { Specificity }=\frac{T N}{F P+T N} \\
& \text { Accuracy }=\frac{T P+T N}{\text { Total population }}
\end{aligned}
$$

Where, $\mathrm{TP}=$ True positive $(\mathrm{AD}$ predicted as $\mathrm{AD}), \mathrm{TN}=$ True negative (Control predicted as control), FP= False positive (Control predicted as $\mathrm{AD}$ ), $\mathrm{FN}=$ False negative (AD predicted as control).

The performance of atrophy, LESH and fusion of atrophy and LESH feature with SVM-GRBF has been depicted in Figure 8 and Figure 9. The true positive, true negative, false positive, false negative, accuracy, sensitivity and specificity obtained with atrophy feature are 48, 45, 5, 2, 93\%, 0.96 and 0.90 respectively. The true positive, true negative, false positive, false negative, accuracy, sensitivity and specificity obtained with LESH feature are 48, 46, 4, 2, 94\%, 0.96 and 0.92 respectively. The true positive, true negative, false positive, false negative, accuracy, sensitivity and specificity obtained with fused feature are 48, 50, 0, 2, 98\%, 0.96 and 1 respectively.

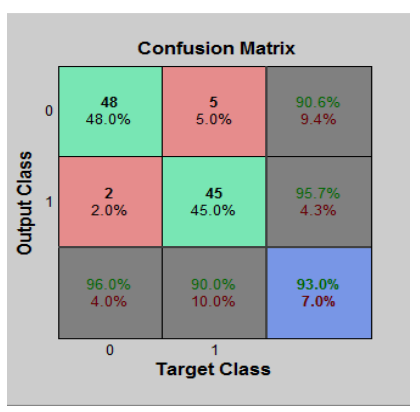

(a)

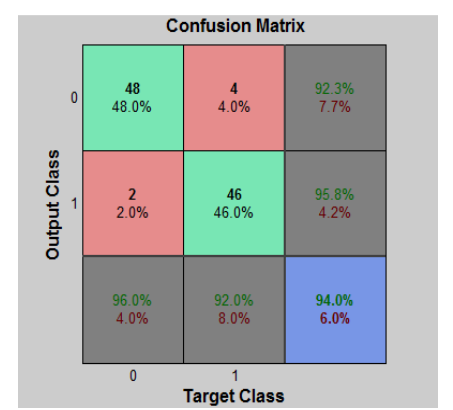

(b)

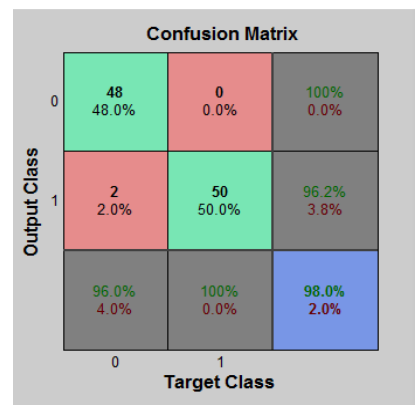

(c)

Figure 8: Performance Measure using Confusion Matrix for SVM-GRBF with (a) Atrophy, (b) LESH and (c) Fused feature

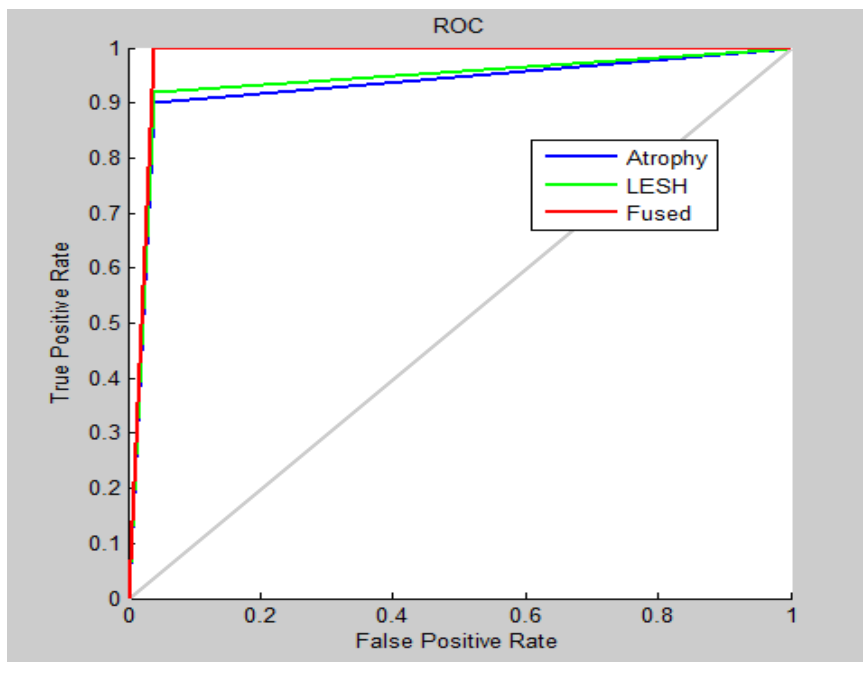

Figure 9: ROC Considering Atrophy, LESH and Fused Feature for SVM-GRBF 
Table 1: Results obtained for SVM-GRBF

\begin{tabular}{|c|c|c|c|c|c|c|c|}
\hline & TP & TN & FP & FN & $\begin{array}{c}\text { Accuracy } \\
(\boldsymbol{\%})\end{array}$ & Sensitivity & Specificity \\
\hline $\begin{array}{c}\text { ATROPHY OF } \\
\text { HIPPOCAMPUS }\end{array}$ & 48 & 45 & 5 & 2 & 93 & 0.96 & 0.90 \\
\hline $\begin{array}{c}\text { SHAPE OF } \\
\text { HIPPOCAMPUS }\end{array}$ & 48 & 46 & 4 & 2 & 94 & 0.96 & 0.92 \\
\hline FUSION & 48 & 50 & 0 & 2 & 98 & 0.96 & 1 \\
\hline
\end{tabular}

Table 2: Comparison With Some Existing Techniques

\begin{tabular}{|c|c|c|c|c|}
\hline AUTHOR & METHOD & DATASET & FEATURES & ACCURACY \\
\hline $\begin{array}{l}\text { I. Beheshti et. al, } \\
\text { (2015)[19] }\end{array}$ & SVM-linear & ADNI & Gray matter loss & $89.65 \%$ \\
\hline $\begin{array}{l}\text { O. B. Ahmed et. al, } \\
\text { (2015)[20] }\end{array}$ & SVM-RBF & $\begin{array}{l}\text { ADNI } \\
\text { Bordeaux-3 } \\
\quad \text { city }\end{array}$ & $\begin{array}{c}\text { Local features of } \\
\text { hippocampus and } \\
\text { posterior cingulated } \\
\text { cortex }\end{array}$ & $\begin{array}{c}\text { ADNI: } 83.77 \% \\
\text { Bordeaux-3 city: } \\
78 \%\end{array}$ \\
\hline $\begin{array}{l}\text { L. Sorensen et. al, } \\
(2015)[21]\end{array}$ & SVM-RBF & $\begin{array}{l}\text { ADNI, AIBL, } \\
\text { Metropolit }\end{array}$ & Hippocampal texture & $\begin{array}{c}\text { ADNI- AUC* : } \\
0.74 \\
\text { AIBL- AUC* : } \\
0.83\end{array}$ \\
\hline $\begin{array}{l}\text { I. Beheshti et. al, } \\
\text { (2016)[22] }\end{array}$ & SVM-linear & ADNI & Gray matter volume & $92.48 \%$ \\
\hline $\begin{array}{l}\text { J. Zhang et. al, } \\
\text { (2017) [23] }\end{array}$ & SVM-linear & ADNI & $\begin{array}{l}\text { Landmark based } \\
\text { feature }\end{array}$ & $88.30 \%$ \\
\hline $\begin{array}{l}\text { L. Sorensen et. al, } \\
\text { (2017) [25] }\end{array}$ & LDA & ADNI, AIBL & $\begin{array}{l}\text { Cortical thickness, } \\
\text { hippocampal shape, } \\
\text { texture and volume }\end{array}$ & $62.7 \%$ \\
\hline $\begin{array}{l}\text { D. Baskar et. } \\
\text { al,(2018)[24] }\end{array}$ & $\begin{array}{c}\text { Kernel fuzzy } \\
\text { c-means }+ \\
\text { back } \\
\text { propagation } \\
\text { ANN }\end{array}$ & $\begin{array}{l}\text { ADNI, } \\
\text { Bordeaux } \\
\text { 3-city }\end{array}$ & $\begin{array}{l}\text { Texture and shape of } \\
\text { hippocampus and } \\
\text { posterior cingulate } \\
\text { cortex }\end{array}$ & $97.63 \%$ \\
\hline $\begin{array}{l}\text { M. Amin-Naji et. } \\
\text { al,(2019)[26] }\end{array}$ & $\begin{array}{l}\text { Convolution- } \\
\text { al neural } \\
\text { network }\end{array}$ & OASIS & - & $98.72 \%$ \\
\hline $\begin{array}{l}\text { U. R. Acharya et. } \\
\text { al,(2019) [27] }\end{array}$ & $\mathrm{k}-\mathrm{NN}$ & private & $\begin{array}{l}\text { Contourlet transform } \\
\text { features }\end{array}$ & $94.54 \%$ \\
\hline $\begin{array}{l}\text { K. Shankar et. al, } \\
\text { (2019)[34] }\end{array}$ & $\begin{array}{l}\text { Convolution- } \\
\text { al neural } \\
\text { network }\end{array}$ & ADNI & Texture feature & $96.23 \%$ \\
\hline $\begin{array}{l}\text { H. Elshatoury et. al, } \\
\text { (2019)[35] }\end{array}$ & SVM & ADNI & Histogram & $69.5 \%$ \\
\hline $\begin{array}{l}\text { K. R. Kruthika et. } \\
\text { al, (2018)[36] }\end{array}$ & $\begin{array}{c}\text { Multistage } \\
\text { classifier } \\
\text { Naive Bayes, } \\
\text { KNN, SVM }\end{array}$ & ADNI & $\begin{array}{l}\text { Cortical thickness, } \\
\text { structural volume }\end{array}$ & $82.3 \%$ \\
\hline Proposed & SVM-GRBF & ADNI & $\begin{array}{c}\text { Atrophy of } \\
\text { hippocampus }\end{array}$ & $93 \%$, \\
\hline Proposed & SVM-GRBF & ADNI & $\begin{array}{c}\text { Shape of } \\
\text { hippocampus(LESH) }\end{array}$ & $94 \%$, \\
\hline Proposed & SVM-GRBF & ADNI & $\begin{array}{c}\text { Fusion of Atrophy and } \\
\text { LESH }\end{array}$ & $98 \%$, \\
\hline
\end{tabular}

*AUC=Area under the receiver operating characteristics curve 
The results obtained with SVM-GRBF have been given in Table 1.A comparison of the present work with some existing works on AD has been given in Table 2. The existing works that have been compared with the present work contains different methods, dataset and features. Most of the works have been performed on ADNI dataset. It is observed from Table 1 that most of the works on $\mathrm{AD}$ have been performed using SVM and ADNI data. Table 1 shows that convolutional neural network is a good classifier for AD detection. SVM has been used in the present study because of its low computational complexity and memory efficiency. In this research work, focus has been given to obtain good classification accuracy with SVM considering atrophy and shape of hippocampus. Size of dataset also influences for considering SVM.

\section{CONCLUSION}

In this work an automatic approach for segmentation of hippocampus from brain MRI has been analyzed. The hippocampus segmentation has shown good results on ADNI data considering $400 \mathrm{~T} 1$ weighted coronal MRI. Atrophy feature has been analyzed on segmented hippocampus. The experiment has shown that the atrophy in AD hippocampus is very high compared to control MRI. We have also experimented LESH feature for analysis of hippocampus shape as it has shown good results in other shape oriented application areas. The LESH feature also gives the difference in between the shape of hippocampus of $\mathrm{AD}$ and control MRI due to shrinkage. The obtained feature vectors have been fed to the SVM-GRBF classifier. The atrophy feature has given 0.96 sensitivity, 0.90 specificity and $93 \%$ accuracy, LESH feature has given 0.96 sensitivity, 0.92 specificity and $94 \%$ accuracy and fusion of the atrophy and shape feature has given 0.96 sensitivity, 1 specificity and $98 \%$ accuracy. Further the work can be improved with combination of other classifier.

\section{REFERENCES}

[1] T. C. Owen, A. A. Howard, W. D. Simon, et al. Atlas-based hippocampus segmentation in Alzheimer's disease and mild cognitive impairment, Neuroimage, Vol. 27, pp. 979-990, 2005.

[2] M. Chupin, A. Hammers, E. Bardinet, et al. Fully Automatic Segmentation of the Hippocampus and the Amygdala from MRI Using Hybrid Prior Knowledge, in Proc. Medical Image Computing and Computer-Assisted Intervention - MICCAI, Brisbane, Australia, 2007, pp. 875-882.

[3] F. V. D. Lijn, T. D. Heijer, M. M. B. Breteler, et al. Hippocampus segmentation in MR images using atlas registration, voxel classification, and graph cuts, Neuroimage, Vol. 43, pp. 708-720, 2008.

[4] K. Kwak, U. Yoon, D-K. Lee, et al. Fully-automated approach to hippocampus segmentation using a graph-cuts algorithm combined with atlas-based segmentation and morphological opening, Magnetic Resonance Imaging , Vol. 31, pp. 1190-1196, 2013.
[5] J. E. Iglesias, J. C. Augustinack, K. Nguyen, et al. A computational atlas of the hippocampal formation using ex vivo, ultra-high resolution MRI: Application to adaptive segmentation of in vivo MRI, Neuroimage , Vol. 115, pp. 117-137, 2015.

[6] P. Aljabar, R. A. Heckemann, A. Hammers, et al. Multi-atlas based segmentation of brain images: Atlas selection and its effect on accuracy, Neuroimage, Vol.46, pp. 726-738,2009.

[7] H. Wang, J. W. Suh, S. Das, et al. Regression-Based Label Fusion for Multi-Atlas Segmentation, in Proc. Computer Vision and Pattern Recognition (CVPR) conference, Colorado, USA. : Colorado Springs, June 2011, pp. 1113-1120.

[8] A. Ghanei, S. H. Zadeh, \& P. J. Windha. Segmentation of the hippocampus from brain MRI using deformable contours, Computerized Medical Imaging and Graphics, Vol. 22, pp. 203-216,1998.

[9] D. Shen, S. Moffat, S. M. Resnick, et al. Measuring Size and Shape of the Hippocampus in MR Images Using a Deformable Shape Model, NeuroImage, Vol. 15, pp. 422-434, 2002.

[10] P. Mesejo, R. Ugolotti, F. D. Cunto, et al. Automatic hippocampus localization in histological images using Differential Evolution-based deformable models, Pattern Recognition Letters, Vol. 34, pp. 299-307, 2013.

[11] M. Kass, A. Witkin, \& D. Terzopoulos. Snakes: active contour models, International Journal of Computer Vision, Vol. 1 (4), pp. 321-331,1988.

[12] T. F. Cootes, C. J. Taylor, D. H. Cooper, et al. Active shape models-their training and application, Computer Vision and Image Understanding, Vol. 61 (1), pp. 38-59, 1995.

[13] T. F. Cootes, G. J. Edwards, \& C. J. Taylor. Active appearance models, IEEE Transactions on Pattern Analysis and Machine Intelligence, Vol. 23 (6), pp. 681-685, 2001.

[14] S. Hu, P. Coupe, J. C. Pruessner, et al. Appearance-based modeling for segmentation of hippocampus and amygdala using multi-contrast MR imaging, NeuroImage, Vol. 58, pp. 549-559, 2011.

[15] M. Chupin, E. Gerardin, R. Cuingnet, et al. Full Automatic Hippocampus Segmentation and Classification in Alzheimer's Disease and Mild Cognitive Impairment Applied on Data From ADNI, Hippocampus, Vol. 19, pp. 579-587,2009.

[16] S. Powell, V. A. Magnotta, H. Johnson, et al. Registration and machine learning-based automated segmentation of subcortical and cerebellar brain structures, NeuroImage, Vol. 39, pp. 238-247, 2008.

[17] S. Tangaro, N. Amoroso, M. Boccardi, et al. Automated voxel-by-voxel tissue classification for hippocampal segmentation: Methods and validation, Physica Medica, Vol. 30, pp. 878-887, 2014.

[18] Y. Chen, B. Shi, Z. Wang, et al. Hippocampus segmentation through multi-view ensemble convnets, in Proc. 2017 IEEE 14th International Symposium on Biomedical Imaging (ISBI 2017), Melbourne, VIC, pp. 192-196. 
[19] I. Beheshti \& H. Demirele. Probability distribution function-based classification of structural MRI for the detection of Alzheimer's disease, Computers in Biology and Medicine, Vol. 64, pp. 208-216, 2015.

[20] O. B. Ahmed, M. Mizotin, J. Benois-Pineau, et al. Alzheimer's disease diagnosis on structural MR images using circular harmonic functions descriptors on hippocampus and posterior cingulate cortex, Computerized Medical Imaging and Graphics , Vol. 44, pp. 13-25, 2015.

[21] L. Sorensen, C. Igel, N. Liv Hansen, et al. Early detection of Alzheimer's disease using MRI hippocampal texture, Human Brain Mapping, Vol. 37, pp. 1148-1161, 2015.

[22] I. Beheshti, H. Demirel, F. Farokhian, et al. Structural MRI-based detection of Alzheimer's disease using feature ranking and classification error, Computer methods and programs in biomedicine, Vol. 137, pp. 177-193, 2016.

[23] J. Zhang, M. Liu, Le An, et al. Alzheimer's Disease Diagnosis Using Landmark-Based Features From Longitudinal Structural MR Images, IEEE Journal of Biomedical and Health Informatics, Vol. 21, pp. 1607-1616, 2017.

[24] D. Baskar, V. S. Jayanthi \& A. N. Jayanthi. An efficient classification approach for detection of Alzheimer's disease from biomedical imaging modalities, Multimedia Tools and Applications (doi:10.1007/s11042-018-6287-8), 2018.

[25] L. Sorensen, C. Igel, A. Pai, et al. Differential diagnosis of mild cognitive impairment and Alzheimer's disease using structural MRI cortical thickness, hippocampal shape, hippocampal texture, and volumetry, NeuroImage: Clinical, Vol. 13 , pp. 470-482, 2017.

[26] M. Amin-Naji, H. Mahdavinataj \& A. Aghagolzadeh Alzheimer's disease diagnosis from structural MRI using Siamese convolutional neural network, in Proc. 4th International Conference on Pattern Recognition and Image Analysis (IPRIA), Tehran, Iran, March 2019, (p. doi:10.1109/pria.2019.8786031).

[27] U. R. Acharya, S. L. Fernandes, J. E. WeiKoh, et al. Automated Detection of Alzheimer's Disease Using Brain MRI Images- A Study with Various Feature Extraction Techniques, Journal of Medical Systems , Vol. 43, pp. 302, 2019.

[28] A. M. Reza. Realization of the Contrast Limited Adaptive Histogram Equalization (CLAHE) for Real-Time Image Enhancement, Journal of VLSI Signal Processing, Vol. 38, pp. 35-44, 2004.

[29] M. C. Morrone \& R. Owens. Feature detection from local energy, Pattern Recognition Letters, Vol. 1, pp. 103-113, 1987.

[30] P. D. Kovesi. Image features from phase congruency, Videre: Journal of Computer Vision Research , MIT Press 1, Vol. 3, pp. 1-26, 1999.

[31] U. Zakir, I. Zafar \& A. E. Edirisinghe. Road sign detection and recognition by using local energy based shape histogram (LESH), International Journal of Image Processing, Vol. 4, pp. 566-582, 2011.
[32] B. Zhang, S. Shan, X. Chen, et al. Histogram of Gabor phase pattern (HGPP): A novel object representation approach for face recognition, IEEE Transactions on Image Processing, Vol. 16(1), pp. 57-68, 2007.

[33] P. D. Kovesi. Phase congruency: A low-level image invariant, Psychological Research, Vol. 64, pp. 136-148, 2000.

[34] K. Shankar, S. K. Lakshmanaprabu, A. Khanna, et al. Alzheimer detection using Group Grey Wolf Optimization based features with convolutional classifier, Computers and Electrical Engineering, Vol. 77, pp. 230-243, 2019.

[35] H. Elshatoury, E. Avots \& G. Anbarjafari. Volumetric Histogram-Based Alzheimer's Disease Detection Using Support Vector Machine, Journal of Alzheimer's Disease, DOI 10.3233/JAD-190704, IOS Press, 2019.

[36] K. R. Kruthika, Rajeswari \& H. D. Maheshappa. Multistage classifier-based approach for Alzheimer's disease prediction and retrieval. Informatics in Medicine Unlocked, doi.org/10.1016/j.imu.2018.12.003, 2018.

[37] T. Prabhakar \& S. Poonguzhali. A Comparison of Classification Methods to Classify the Breast Lesions from Ultrasound Images using Texture Feature Extraction, International Journal of Advanced Trends in Computer Science and Engineering, Vol. 9(5), pp. 6877-6880, 2020. 